\title{
Preface to Topical Issue: Recalibration of the Sunspot Number
}

\author{
F. Clette ${ }^{1}(D) \cdot$ E.W. Cliver ${ }^{2} \cdot$ L. Lefèvre ${ }^{1} \cdot$ L. Svalgaard ${ }^{3}$. \\ J.M. Vaquero ${ }^{4} \cdot$ J.W. Leibacher ${ }^{5}$
}

Published online: 17 November 2016

(C) Springer Science+Business Media Dordrecht 2016

\begin{abstract}
This topical issue contains articles on the effort to recalibrate the sunspot number (SN) that was initiated by the Sunspot Number Workshops. These workshops led to a revision of the Wolf sunspot number (WSN) and a new construction of the group sunspot number (GSN), both published herein. In addition, this topical issue includes three independently proposed alternative SN time series (two Wolf and one group), as well as articles providing historical context, critical assessments, correlative analyses, and observational data, both historical and modern, pertaining to the sunspot-number time series. The ongoing effort to understand and reconcile the differences between the various new sunspot number series is briefly discussed.
\end{abstract}

\section{Introduction}

The sunspot number ( $\mathrm{SN}$ ) is arguably the most intensely used time series in all of astrophysics (Charbonneau, 2010) and has been identified as the "longest-running scientific experiment" (Owens, 2013). Over the last decade, more than one hundred scientific articles per year on average were based on the sunspot number in the fields of solar and solar-terrestrial physics alone.

In solar physics, the multi-century sunspot series serves as a primary constraint on solardynamo models. It also provides a quantitative reference for past solar-irradiance and solarwind reconstructions and for the calibration of cosmogenic-isotope records, which then give

Sunspot Number Recalibration

Guest Editors: F. Clette, E.W. Cliver, L. Lefèvre, J.M. Vaquero, and L. Svalgaard

F. Clette

frederic.clette@oma.be

1 World Data Center SILSO, Observatoire Royal de Belgique, Brussels, Belgium

2 National Solar Observatory, Boulder, CO, USA

3 W.W. Hansen Experimental Physics Laboratory, Stanford University, Stanford, CA, USA

4 Departamento de Física, Universidad de Extremadura, Mérida, Spain

5 National Solar Observatory, Tucson, AZ, USA 


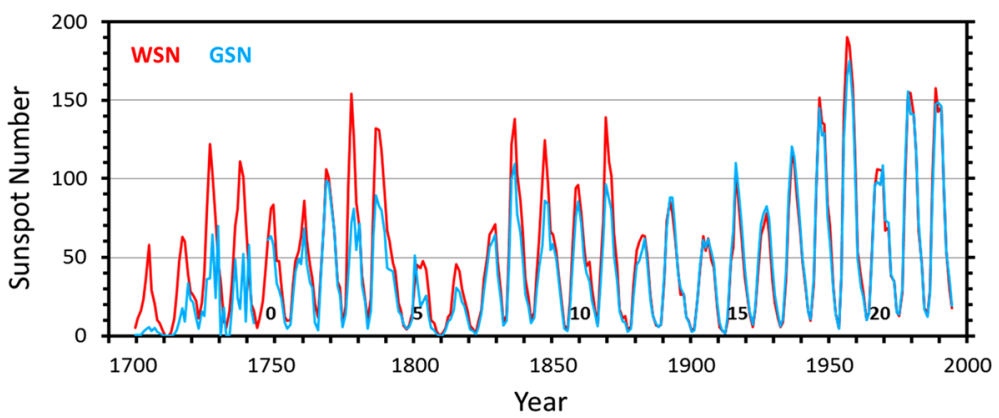

Figure 1 Comparison of yearly mean values of the Wolf (WSN) and Group (GSN) sunspot-number time series. Cycle numbers are given at the bottom of the panel.

access to the evolution of solar activity over millennia. Beyond research, the sunspot has operational space-weather applications (e.g. satellite drag, radio communication, groundinduced current effects on power grids and pipelines). Finally, next to a few other icons of astronomy such as Saturn's rings, the Milky Way, and black holes, sunspots are part of the general culture.

Wolf $(1851,1856)$ defined the sunspot number (WSN) as a function of the daily number of spot groups and individual spots on the Sun. Wolf's definition held for $\approx 150$ years until it was challenged by the solar-activity time series of Hoyt and Schatten (1998a, 1998b) that was based solely on the daily count of sunspot groups. The group sunspot number (GSN) was significantly lower $(\approx 30 \%)$ on average than the WSN before $\approx 1885$ (Figure 1$)$. Suddenly there were two sunspot numbers with no consensus as to which more accurately described our star's behavior. Although this discrepancy was pointed out by Hoyt and Schatten at the time, no significant investigation of its origin was undertaken for more than a decade. In the absence of evidence to determine which of the series was more reliable, one or the other series was simply used as the standard reference in different publications. The GSN was often preferred because it was the longest series and the only one that included the last Grand Minimum of solar activity (Maunder Minimum).

To address this unacceptable situation, a community-wide series of sunspot-number workshops was initiated by F. Clette, E. Cliver, and L. Svalgaard in mid-2011 (Cliver, Clette, and Svalgaard, 2013; Cliver et al., 2015; Clette et al., 2014). This initiative resulted from the fortuitous coincidence of focused calls from E. Cliver and L. Svalgaard to the World Data Center (WDC) for the Sunspot Index (then named SIDC) and new objectives defined for the SIDC by F. Clette, who became its director in April 2011 and had begun to document and critically review the data and methods used for the production of the Zürich and Brussels sunspot number. Four workshops were held successively at Sunspot, NM, USA in September 2011, Brussels, Belgium in May 2012 (Figure 2), Tucson, AZ, USA in January 2013, and Locarno, Switzerland in May 2014. In July 2015, the revised WSN (Clette et al., 2015; Clette and Lefèvre, 2016), designated $S_{\mathrm{N}}$, was officially published on the website of the WDC for the Sunspot Index and Long-term Solar Observations (SILSO) at the Royal Observatory of Belgium: www.sidc.be/silso/. This revised series marked the first end-to-end revision of the sunspot number since its creation by R. Wolf in 1849. Simultaneously, an independent construction of a group-count series (Svalgaard and Schatten, 2016), that - upon scaling - closely agreed with the $S_{\mathrm{N}}$, was released.

The announcement of the $S_{\mathrm{N}}$ time series prompted the publication of two new alternative sunspot time series: one Wolf-type formulation (Friedli 2016, 2017) and one group- 


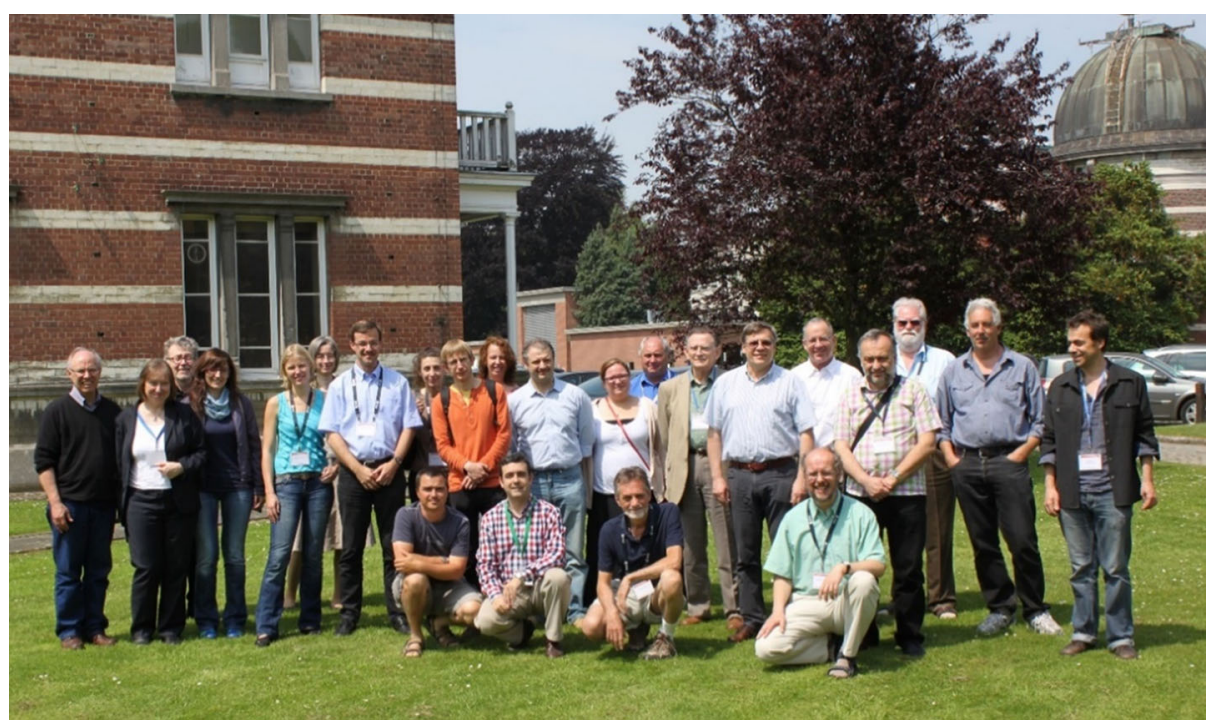

Figure 2 Participants, Second SN Workshop, Royal Observatory of Belgium, Brussels, 21 - 25 May 2012. Bottom row, left to right: W. Pötzi, J. Vaquero, R. Howe, F. Clette. Top row, left to right: E. Cliver, L. van Driel-Gesztelyi, K. Mursula, M. Laurenza, I. Cnossen, N. Crosby, T. Friedli, G. de Toma, R. Arlt, L. Wauters, I. Usoskin, L. Lefèvre, A. Tlatov, D. Willis, A. Pevtsov, H. Hudson, R. Brajša, L. Svalgaard, L. Bertello, M. Kretzschmar.

based series (Usoskin et al., 2016), which are presented in this topical issue. Earlier, Lockwood, Owens, and Barnard (2014) and Lockwood et al. (2016b, 2016d) had proposed a revision/extension of the original Wolf series that differs from that of Clette and Lefèvre (2016). The introduction of these five new series signaled a new era in sunspot-number research.

This topical issue collects 36 articles documenting the analyses pertaining to this resurgence of interest in the sunspot number. In addition to the various new SN time series, these 36 articles include a historical review, compilations of observations, critiques of old and new sunspot-number time series, correlative analyses, and documentation of both historical and modern sunspot observations.

\section{Organization and Brief Commentary}

This topical issue is broken into five sections:

i) Historical Context and Data (8 articles)

ii) New Time Series (5 articles)

iii) Critiques (9 articles)

iv) Correlates (9 articles)

v) Modern Sunspot Observations (5 articles)

In Section i), Stenflo (2016) gives a fascinating personal account of the circumstances attending the transfer of the responsibility for constructing the official sunspot number from Zürich to Brussels in 1980. The next seven articles deal with historical sunspot observations. 
Arlt and Vaquero are the acknowledged leaders in this field. Arlt and collaborators have digitized and analyzed the sunspot records of Staudach (Arlt, 2008), Schwabe (Arlt, 2011; Arlt et al., 2013), and Spörer (Diercke, Arlt, and Denker, 2015). In this section, Vaquero and colleagues have contributions on the sunspot observations of Flamsteed during the Maunder Minimum (Carrasco and Vaquero, 2016), as well as those from the Ebro Observatory from 1910 - 2014 (Curto et al., 2016), and by Aguilar for 1914-1920 (Lefèvre et al., 2016). Willis, Wild, and Warburton (2016) and Willis et al. (2016) examine the records for the early years (1874-1885) of the Royal Greenwich Observatory sunspot patrol in detail, as does Friedli (2016) for Rudolf Wolf's (1849 - 1876) sunspot observations. Svalgaard (2017) argues that modern group-splitting will increase Staudach's 1749-1799 sunspot group counts by $25 \%$ on average.

Section ii) contains the new time series of Clette and Lefèvre (2016) and Svalgaard and Schatten (2016) as well as the independently derived series of Usoskin et al. (2016) and Friedli (2016, 2017). While the Clette and Lefèvre (2016) and Svalgaard and Schatten series hew more closely to the original WSN than do the Hoyt and Schatten (1998a, 1998b) GSN, the series of Usoskin et al. (2016) and Friedli (2017), as well as that of Lockwood, Owens, and Barnard (2014) and Lockwood et al. (2016b, 2016d), are more closely aligned with the GSN. Also in this section, Dudok de Wit, Lefèvre, and Clette (2016) provide a first determination of uncertainties in the WSN series, with new insights about the temporal variations of these uncertainties.

Section iii) contains critiques and corrections of the various new and old sunspot-number time series, mostly focusing on specific inhomogeneities and time intervals. Clette et al. (2016) report on the effect of the irregular drift in the sunspot counts of the Locarno reference station that began in 1982 shortly after the transition from Zürich to Brussels. Cliver and Ling (2016) examine the original Hoyt and Schatten (1998a, 1998b) time series and conclude that the lower values of the GSN relative to the WSN before $\approx 1885$ are primarily due to an inhomogeneity from 1874-1915 in the Royal Greenwich Observatory (RGO) record of sunspot groups that Hoyt and Schatten used as their reference observer ( $c f$. Willis et al., 2016). During the sunspot-number workshops, Svalgaard for the first time brought the sunspot weighting that was apparently instituted by Waldmeier in 1947 to the general attention of the solar community. Svalgaard, Cagnotti, and Cortesi (2017) assess the effect of that weighting on the post-1946 WSN series. In a series of articles in this topical issue (Lockwood, Owens, and Barnard, 2016; Lockwood et al., 2016a, 2016b, 2016c) and elsewhere (Lockwood et al., 2016d), Lockwood and colleagues i) use comparisons with ionospheric, geomagnetic, and auroral data to question the amplitude of the 1947 scale jump in the WSN time series, and ii) challenge the validity of the standard-observer normalization procedure used by Svalgaard and Schatten (2016) and also by Hoyt and Schatten (1998a, 1998b), which relies on linear-regression comparisons of secondary observers with a standard observer based on the complete interval of observer overlap (see also Usoskin, Kovaltsov, and Chatzistergos, 2016). Zolotova and Ponyavin $(2016)^{1}$ question the extreme low values of the GSN during the Maunder Minimum ( $c f$. Usoskin et al., 2015). In a comparison of several of the new and old sunspot-number time series, Cliver (2016) points out incongruities in the normalization scheme of the Usoskin et al. (2016) GSN series and a lack of fidelity between that $\mathrm{SN}$ series and a recent construction of the long-term solar-wind magnetic-field strength (Owens et al., 2016).

Section iv) contains correlations between sunspot-number time series and other solaractivity measures. These include sunspot area (Li et al., 2016; Carrasco et al., 2016a;

\footnotetext{
${ }^{1}$ We are saddened to report the passing of Dmitri Ponyavin on 8 October 2016.
} 
Muraközy, Baranyi, and Ludmány, 2016), total solar irradiance (Kopp et al., 2016), Ca II K index (Bertello et al., 2016), extreme ultra-violet flux (Svalgaard, 2016), X-ray flare and CME rates (Winter, Pernak, and Balasubramaniam, 2016), solar-modulation potential derived from cosmogenic nuclides (Muscheler et al., 2016), and solar-cycle timing characteristics (Carrasco et al., 2016b).

Section v) contains articles on modern sunspot-number observations. The first article in this section, by Vaquero et al. (2016), represents one of the key outputs of the sunspotnumber workshops: the revised Hoyt and Schatten (1998a, 1998b) group sunspot number data base (1610 - present). This section also contains valuable reports from the longstanding observatories at Locarno (since 1957; Cortesi et al., 2016), Debrecen (since 1958; Baranyi, Gyori, and Ludmány, 2016), and Kanzelhöhe (since 1943; Pötzi et al., 2016), as well as a description of the $\approx$ ten-year record (2003 -2012) of sunspot numbers obtained by the ISOON telescope (Balasubramaniam and Henry, 2016).

A list of all of the articles in this topical issue, organized by section, is given in the Table of Contents section of the References.

\section{A Look Ahead}

This topical issue signals a new phase in sunspot and solar-cycle research. The multiple alternate versions of the sunspot-number series published recently, including those in this topical issue, were an unexpected consequence of the sunspot-number workshops, and attest to a growing level of interest in long-term solar and solar-terrestrial studies, i.e. space climate (Mursula, Usoskin, and Cliver, 2004).

This recent rapid evolution marks a deeper transition, beyond the simple change in the sunspot numbers. The very nature of the series has evolved from a static "heritage" series produced by a single institution to a modern dynamic dataset. Just as any modern solar data series, it is now open to future advances, and all parts of the series are subject to future upgrades, incorporating newly recovered input data or new state-of-the-art processing methods. Moreover, while in the past the sunspot number was almost exclusively built internally by a single data center, current and future revisions will be based on published results from various scientific teams, working in conjunction with WDC-SILSO.

The lack of current consensus on the correct version of the sunspot number is explicit in the articles in Section iii) of this topical issue. The shared view among the involved scientists is that the disparity evident between the two current groupings of sunspot numbers (Clette and Lefèvre (2016) and Svalgaard and Schatten (2016), on the one hand, and Usoskin et al. (2016), Lockwood, Owens, and Barnard (2014), Lockwood et al. (2016c), and Friedli (2017), on the other) is as unacceptable as the discrepancy between the original WSN and GSN time series.

Thus an effort has been initiated, akin to the original sunspot-number workshops, to understand and reconcile the differences between the various sunspot-number time series in order to obtain a single, community-wide vetted, and accepted sunspot number. This actually means two time series, one as defined by Wolf, and a separate group number. The two series complement each other; the GSN can be extended farther back in time to cover the important Maunder Minimum period, while the WSN formulation provides detailed information on group size.

Despite their differences in definition, we expect the two resultant time series to be highly correlated. If the past is any guide, this reconciliation process will take time, an estimated two to three years, with provision (as was made for $S_{\mathrm{N}}$; Clette et al., 2015; Clette and 
Lefèvre, 2016) for subsequent updated versions in the longer term, as more historical data are discovered and processed and our understanding of the sunspot number as a measure of solar activity grows.

The once staid sunspot number, long-viewed as an unquestionable time series in solarterrestrial physics - before giving way to a decade in which two disparate series coexisted - has become the focus of intense investigation. Given the far-reaching importance of this time series, we believe that such attention is long overdue.

Acknowledgments We thank the amateur astronomers, who as an avocation have recorded sunspots over the past four centuries, thereby making the sunspot number a robust time series. In particular, we are grateful to Jan Alvestad, Andreas Bulling, Thomas Friedli, and Rodney Howe for their active participation in the sunspot-number workshops and all currently active observers (amateur and professional) contributing to the worldwide WDC-SILSO network.

Frédéric Clette and Laure Lefèvre acknowledge material support by the hosting institute of the WDCSILSO since 1981, the Royal Observatory of Belgium, and financial support by the Belgian Solar-Terrestrial Center of Excellence (STCE) since 2005, through its current director, Ronald Van der Linden. Frédéric Clette would like to thank the key people who contributed to the recent modernization of the World Data Center: Olivier Lemaître, David Berghmans, Olivier Boulvin, and Sarah Willems.

\section{References}

\section{In Preface}

Arlt, R.: 2008, Digitization of sunspot drawings by Staudacher in 1749-1796. Solar Phys. 247, 399. DOI.

Arlt, R.: 2011, The sunspot observations by Samuel Heinrich Schwabe. Astron. Nachr. 332, 805. DOI.

Arlt, R., Leussu, R., Giese, N., Mursula, K., Usoskin, I.G.: 2013, Sunspot positions and sizes for $1825-1867$ from the observations by Samuel Heinrich Schwabe. Mon. Not. Roy. Astron. Soc. 433, 3165. DOI.

Charbonneau, P.: 2010, Dynamo models of the solar cycle. Living Rev. Solar Phys. 7, 3. DOI.

Clette, F., Svalgaard, L., Vaquero, J.M., Cliver, E.W.: 2014, Revisiting the sunspot number. A 400-year perspective on the solar cycle. Space Sci. Rev. 186, 35. DOI.

Clette, F., Cliver, E.W., Lefèvre, L., Svalgaard, L., Vaquero, J.M.: 2015, Revision of the sunspot number(s). Space Weather 13, 529. DOI.

Cliver, E.W., Clette, F., Svalgaard, L.: 2013, Recalibrating the sunspot number (SSN): the SSN workshops. Cent. Eur. Astrophys. Bull. 37(2), 401. ADS.

Cliver, E.W., Clette, F., Svalgaard, L., Vaquero, J.M.: 2015, Recalibrating the sunspot number (SN): the 3rd and 4th SN workshops. Cent. Eur. Astrophys. Bull. 39, 1. ADS.

Diercke, A., Arlt, R., Denker, C.: 2015, Digitization of sunspot drawings by Spörer made in $1861-1894$. Astron. Nachr. 336, 53. DOI.

Hoyt, D.V., Schatten, K.H.: 1998a, Group sunspot numbers: a new solar activity reconstruction. Solar Phys. 179, 189. DOI.

Hoyt, D.V., Schatten, K.H.: 1998b, Group sunspot numbers: a new solar activity reconstruction. Solar Phys. 181, 491. DOI.

Lockwood, M., Owens, M.J., Barnard, L.: 2014, Centennial variations in sunspot number, open solar flux, and streamer belt width: 2. Comparison with the geomagnetic data. J. Geophys. Res. 119, 5183. DOI.

Lockwood, M., Owens, M.J., Barnard, L., Usoskin, I.G.: 2016d, An assessment of sunspot number data composites over $1845-2014$. Astrophys. J. 824, 54. DOI.

Mursula, K., Usoskin, I., Cliver, E.: 2004, Preface. Solar Phys. 224, 3. DOI.

Owens, B.: 2013, Long-term research: slow science. Nature 495(7441), 300. 21 March 2013, News Feature. DOI.

Owens, M.J., Cliver, E., McCracken, K.G., Beer, J., Barnard, L., Lockwood, M., Rouillard, A., et al.: 2016, Near-Earth heliospheric magnetic field intensity since 1750: 1. Sunspot and geomagnetic reconstructions. J. Geophys. Res. 121, 6048. DOI.

Usoskin, I.G., Kovaltsov, G.A., Chatzistergos, T.: 2016, Dependence of the sunspot-group size on the level of solar activity and its influence on the calibration of solar observers. Solar Phys. 291. DOI.

Usoskin, I.G., Arlt, R., Asvestari, E., Hawkins, E., Käpylä, M., Kovaltsov, G.A., Krivova, N., et al.: 2015, The Maunder minimum (1645-1715) was indeed a grand minimum: a reassessment of multiple datasets. Astron. Astrophys. 581, A95. DOI. 
Wolf, R.: 1851, Sonnenflecken-Beobachtungen in der zweiten Hälfte des Jahres 1850. Mitt. Nat.forsch. Ges. Bern 207, 89.

Wolf, R.: 1856, Mittheilungen über die Sonnenflecken. Astron. Mitt. Eidgenöss. Sternwarte Zür. 1(I), 3.

\section{Table of Contents}

\section{Preface}

Clette, F., Cliver, E.W., Lefèvre, L., Svalgaard, L., Vaquero, J.M., Leibacher, J.W.: 2016, Preface to Topical Issue: Recalibration of the Sunspot Number. Solar Phys. 291. DOI

\section{i) Historical Context and Data}

Stenflo, J.O.: 2016, Transition of the sunspot number from Zurich to Brussels in 1980: a personal perspective. Solar Phys. 291. DOI.

Carrasco, V.M.S., Vaquero, J.M.: 2016, Sunspot observations during the Maunder minimum from the correspondence of John Flamsteed. Solar Phys. 291. DOI.

Svalgaard, L.: 2017, A recount of sunspot groups on Staudach's drawings, Solar Phys. (accepted).

Friedli, T.K.: 2016, Sunspot observations of Rudolf Wolf from 1849-1893. Solar Phys. 291. DOI.

Willis, D.M., Wild, M.N., Warburton, J.S.: 2016, Re-examination of the daily number of sunspot groups for the royal observatory, Greenwich (1874-1885). Solar Phys. 291. DOI.

Willis, D.M., Wild, M.N., Appleby, G.M., Macdonald, L.T.: 2016, The Greenwich Photo-heliographic Results (1874-1885): observing telescopes, photographic processes, and solar images. Solar Phys. 291. DOI.

Curto, J.J., Solé, J.G., Genescà, M., Blanca, M.J., Vaquero, J.M.: 2016, Historical heliophysical series of the Ebro observatory. Solar Phys. 291. DOI.

Lefèvre, L., Aparicio, A.J.P., Gallego, M.C., Vaquero, J.M.: 2016, An early sunspot catalog by Miguel Aguilar for the period $1914-1920$. Solar Phys. 291. DOI.

\section{ii) New Time Series}

Clette, F., Lefèvre, L.: 2016, The new sunspot number: assembling all corrections. Solar Phys. 291. DOI.

Svalgaard, L., Schatten, K.H.: 2016, Reconstruction of the sunspot group number: the Backbone method. Solar Phys. 291. DOI.

Usoskin, I.G., Kovaltsov, G.A., Lockwood, M., Mursula, K., Owens, M., Solanki, S.K.: 2016, A new calibrated sunspot group series since 1749: statistics of active day fractions. Solar Phys. 291. DOI.

Friedli, T.K.: 2017, Homogenization of the Wolf series from 1749 to 2014. Solar Phys. (submitted).

Dudok de Wit, T., Lefèvre, L., Clette, F.: 2016, Uncertainties in the sunspot numbers: estimation and implications. Solar Phys. 291. DOI.

\section{iii) Critiques}

Clette, F., Lefèvre, L., Cagnotti, M., Cortesi, S., Bulling, A.: 2016, The revised Brussels-Locarno sunspot number (1981 - 2015). Solar Phys. 291. DOI.

Cliver, E.W., Ling, A.G.: 2016, The discontinuity circa 1885 in the group sunspot number. Solar Phys. 291. DOI.

Svalgaard, L., Cagnotti, M., Cortesi, S., 2017, The effect of sunspot weighting. Solar Phys. (submitted).

Lockwood, M., Scott, C.J., Owens, M.J., Barnard, L., Willis, D.M.: 2016a, Tests of sunspot number sequences: 1. Using ionosonde data. Solar Phys. 291. DOI.

Lockwood, M., Owens, M.J., Barnard, L., Scott, C.J., Usoskin, I.G., Nevanlinna, H.: 2016b, Tests of sunspot number sequences: 2. Using geomagnetic and auroral data. Solar Phys. 291. DOI.

Lockwood, M., Owens, M.J., Barnard, L., Usoskin, I.G.: 2016c, Tests of sunspot number sequences: 3. Effects of regression procedures on the calibration of historic sunspot data. Solar Phys. 291. DOI.

Lockwood, M., Owens, M.J., Barnard, L.: 2016, Tests of sunspot number sequences: 4. Discontinuities around 1946 in various sunspot number and sunspot group number reconstructions. Solar Phys. 291. DOI.

Zolotova, N.V., Ponyavin, D.I.: 2016, How deep was the Maunder minimum? Solar Phys. 291. DOI.

Cliver, E.W.: 2016, Comparison of new and old sunspot number time series. Solar Phys. 291. DOI. 


\section{iv) Correlates}

Li, K.J., Zhang, J., Feng, W., Li, F.Y.: 2016, Investigation of sunspot area varying with sunspot number. Solar Phys. 291. DOI.

Carrasco, V.M.S., Vaquero, J.M., Gallego, M.C., Sánchez-Bajo, F.: 2016a, A normalized sunspot-area series starting in 1832: An update. Solar Phys. 291. DOI.

Muraközy, J., Baranyi, T., Ludmány, A.: 2016, An alternative measure of solar activity from detailed sunspot datasets. Solar Phys. 291. DOI.

Kopp, G., Krivova, N., Wu, C.J., Lean, J.: 2016, The impact of the revised sunspot record on solar irradiance reconstructions. Solar Phys. 291. DOI.

Bertello, L., Pevtsov, A., Tlatov, A., Singh, J.: 2016, Correlation between sunspot number and Ca II K emission index. Solar Phys. 291. DOI.

Svalgaard, L.: 2016, Reconstruction of solar extreme ultraviolet flux 1740-2015. Solar Phys. 291. DOI.

Winter, L.M., Pernak, R.L., Balasubramaniam, K.S.: 2016, Comparing SSN index to X-ray flare and coronal mass ejection rates from solar cycles $22-24$. Solar Phys. 291. DOI.

Muscheler, R., Adolphi, F., Herbst, K., Nilsson, A.: 2016, The revised sunspot record in comparison to cosmogenic radionuclide-based solar activity reconstructions. Solar Phys. 291. DOI.

Carrasco, V.M.S., Aparicio, A.J.P., Vaquero, J.M., Gallego, M.C.: 2016b, The new sunspot number index and solar cycle characteristics. Solar Phys. 291. DOI.

\section{v) Modern Sunspot Observations}

Vaquero, J.M., Svalgaard, L., Carrasco, V.M.S., Clette, F., Lefèvre, L., Gallego, M.C., Arlt, R., Aparicio, A.J.P., Richard, J.-G., Howe, R.: 2016, A revised collection of sunspot group numbers. Solar Phys. 291. DOI.

Cortesi, S., Cagnotti, M., Bianda, M., Ramelli, R., Manna, A.: 2016, Sunspot observations and counting at Specola Solare Ticinese in Locarno since 1957. Solar Phys. 291. DOI.

Baranyi, T., Gyori, L., Ludmány, A.: 2016, On-line tools for solar data compiled at the Debrecen Observatory and their extensions with the Greenwich sunspot data. Solar Phys. 291. DOI.

Pötzi, W., Veronig, A.M., Temmer, M., Baumgartner, D.J., Freislich, H., Strutzmann, H.: 2016, 70 Years of sunspot observations at the Kanzelhöhe Observatory: systematic study of parameters affecting the derivation of the relative sunspot number. Solar Phys. 291. DOI.

Balasubramaniam, K.S., Henry, T.W.: 2016, Sunspot numbers from ISOON: a ten-year data analysis. Solar Phys. 291. DOI. 